\title{
Contemporary Review on Recent Applications of Gold and Silver Nanoparticles as Nanotheranostic Non-viral Tools
}

\author{
${ }^{* 1}$ Kirti Rani \\ ${ }^{1}$ Assistant Professor (III), \\ Amity Institute of Biotechnology, Amity University Uttar Pradesh, Noida, India
}

\begin{abstract}
Nanomaterials are known as chemically or biologically engineered materials having nanodimension of 1-100nm. These have been shown to exhibit various novel properties e.g. enhanced reactivity, greater sensing capability and improved mechanical strength. Various improved procedures are still under innovative considerations to enhance the potency, safety, and efficiency of chosen plasmid DNA vectors to be used as safe vector design to load desired protein and antibiotic resistance genes in the host for their site specific targeted delivery Previously, various improved nano-sized formulations and dispersions have been proposed for drug or gene delivery in major and various established pharmaceutical companies such as colloidal silver nanoparticles, silver nanoparticles and nanoscaffolds. Now these days, nonviral gene/drug therapy is well promising diagnostic and therapeutic modality for the treatment of various genetic, metabolic and neurodegenerative disorders. These nonviral nano-approaches has been found to be an excellent and safe alternative gene transfer vehicles to other popular viral vectors due to having significant favourable properties such as lack of immunogenicity, low toxicity and potential for tissue specificity and targeted drug delivery. Hence, these nonviral nano-clinical and genetic approaches have also been tested in preclinical studies and human clinical trials over the last decades and can be used as more advanced and improved nanotheranostic tools.
\end{abstract}

Keywords: Nanoparicle; Nanobiomaterial; Nanotheranostic tool; Gold nanopaticles; Silver nanoparticles

\section{INTRODUCTION}

Recently, non-viral gene vectors such as lipoplexes, liposomes, polyplexes, and nanoparticles have also been proposed them as nano-sized diagnostic and therapeutic DNA-based nanomedicine into the target cell that can facilitate development of nucleic acids-based nanotherapy as nanotheranostic tool (Pathak et al., 2009). So, designing of new multifunctional nanodevices and nanobiomaterials are become more promising and safer choice for gene delivery over viral vector delivery such as use of E. coli, Lentil virus, Adenovirus, Herpes virus and Retro viruses. Non-viral vectors based gene therapy using cationic lipids or polymers, gold nanoparticles (AuNPs) and sliver nanoparticles (AgNPs) along with their improved nanoconjugates or nanoscaffold were found to have promising safe delivery potential as compared to viral gene delivery system (Katragadda et al., 2012; Ramamoorth et al., 2015). Recently, the gene delivery have been reported by using nano-carriers including calcium phosphates, lipids, and cationic polymers, polyplexes, lipoplexes, lipopolyplexes, chitosan, polyethylenimine, polyamidoamine dendrimers, and poly(lactide-co-glycolide) (Germershaus et al., 2015; Jin et al., 2014). Nanobiomaterials were loaded with desired delivery materials to be used as non-viral gene delivery using polyethylenimine-coated magnetic nanoparticles and cell-penetrating peptides (CPPs) and used their impregnation into host genome (Huang et al., 2015). Nanoparticles gene carrier were developed with ease due to having low toxicity and good biocompatibility that have promising improved transfection efficiency to target the cancer or tumor cells as nonviral gene vector (Blasiack et al., 2013). Nonviral Gene therapy has been recently reported a promising nanotheranostic tool to investigate the improved anticancer efficacy suggesting that nanomedicine provides novel opportunities to safely gene deliver method to treat cancers or tumors (Suna et al., 2014).

\section{Gold NANOPARTICLES (AUNPS)}

Gold nanoparticles were reported to be an attractive nanoscaffold as nontoxic delivery vehicle. It was achieved by decorating the surface of these nano-biovehicles with desired specific antibodies and 


\section{Kirti Rani}

grafting with fabrication of active functional groups (e.g., polyethylene glycol and zwitterionic entities to increase their plasma protein adsorption followed by improving the pharmacokinetics and evading immune surveillance (Ding et al., 2014). Key features of oligonucleotide and small interfering RNA-modified AuNPs conjugates were found to be more promising intracellular gene regulation agents over various conventional agents to activate immune-related genes and pathways in human peripheral blood mononuclear cells, but not an immortalized lineage-restricted cell line. (Kim et al., 2011). In last decade, the potential of double-stranded RNAs to interfere with gene expression has been studied to carry out new nanotheranostic implication and its efficiency was demonstrated to drive chemical and biological approaches for safe, nonpathogenic, self-tracking, and universally valid nanocarrier (Conde et al., 2012). In addition, it was also observed that AuNPs coupled Ad vector system might be considered very potent nanocarrier for simultaneous targeting, imaging, and combined hyperthermia and tumor gene therapy (Saini et al., 2014).

Recently, AuNPs-nanoscaffold was reported an attractive and applicable scaffold for delivery of nucleic acids when used as covalent and noncovalent gold nanoparticle conjugates for applications in gene delivery and RNA-interference technologies including their effective endosomal entrapment/escape and active delivery expression potential of nucleic acids in the host genome (Tiwari et al., 2011; Mieszawska et al., 2013; Yah et al., 2013). The use of partially acetylated dendrimer-entrapped gold nanoparticles (AuDENPs) was also designed for gene delivery applications that may serve as key nonviral gene delivery for safe gene delivery applications with desired gene transfection efficiency (Hou et al., 2015; Hossain et al., 2015). Improved cationic gold nanoparticles were synthesized and its gene expression efficacy were confirmed by fluorescent microscopy and luciferase assay to demonstrate the transgene delivery capability of the ssPEI-GNPs (Uthaman et al., 2015).

Ultra-Small AuNPs (> $2 \mathrm{~nm}$ size) were synthesized and further studied for their potential use of intranucleus delivery nonviral gene delivery vehicle as improved nanotherapeutic device being a nanotheranostic tool. It was investigated for delivery of a triplex-forming oligonucleotide (TFO) that binds to the c-myc promoter. (Huo et al., 2014).

\section{Silver NANOPARTiCles (AgNPs)}

Silver nanoparticles (AgNPs) exhibit versatile properties which endorse their vast spectrum of their applications in nanomedicine. Scientists were found that AgNPs have high antimicrobial efficacy against many pathogenic bacteria species including Escherichia coli, Neisseria gonorrhea, Chlamydia trachomatis and other pathological viruses that can be considered ideal gene delivery systems for tissue regeneration and used as safe biosensors being a nonviral nanotheranostic tool (Marin et al, 2015).

Silver nanoparticle (AgNPs) tagged with photolabile nucleic acid conjugates have been used to perform the inducible gene silencing for delivery of therapeutic agents such as antisense oligonucleotides (Brown et al., 2011) Biofunctionalized stable AgNPs with good DNA binding ability were proposed for efficient transfection and low toxicity to be used in biomedical applications especially in wound healing and cancer gene therapy (Sarkar et al., 2015). Montmorillonite conjugated silver nanoparticles were also used for binding with plasmid pcDNA-GFP to carry out desired a gene delivery (Sironmani et al., 2015). AgNPs as oiligonucleotide-thiol conjugate, poly adenine (polyA) DNA functionalized gold nanoparticles (AuNPs) were fabricated with high density of DNA attachment and high hybridization ability to facilitate the appended recognition for hybridization to demonstrate its ideal tunable plasmonic biosensor. (Lu et al., 2015). Various fabricated AgNPs conjugates were also used as photoactivated antisense mediated nonviral gene delivery vehicle being nontoxic nanotheranostic tool due to having unique photophysical properties of silver that were characterized for their clinical contribution as potential photoactivated drug delivery vectors (Dong et al., 2015).

\section{CONCLUSiON}

So, this very recent mini review article can be quite informative and latest update of nanotheranostic applications of gold nanoparticles (AuNPs) and silver nanoparticles (AgNPs). Previously, number of clinical investigations were carried out on various side effects of viral and non-viral gene delivery vehicles and found their pathogenicity with the time in the targeted host cells. And, it was found that conjugates and nanoscaffolds of nanobiomaterials such as metal, polymers, chitosan etc were less 
toxic and more biocompatible over the viral gene delivery vehicle to be used as nanotheranostic tool. Among them, gold nanoparticles and silver nanoparticles as well as their derivatives or conjugates were found to be reported more safe and popular with the ease of its easy fabrication, high biocompatibility and easy photoluminescent based trafficking in host cell metabolism. Hence, these recent reports are better described the applications of the gold nanoparticles (AuNPs) and silver nanoparticles (AgNPs) being an safe nonviral gene or drug delivery vehicles being more potent nanotheranostic tool.

ACKNOWLEDGMENT: I would like to express my cordially appreciation to Amity University Uttar Pradesh, Noida (INDIA).

\section{REFERENCES}

[1] Blasiack, B.; van Veggeal, F. C. J. M. and Tomanek B. Applications of Nanoparticles for MRI Cancer Diagnosis and Therapy. J Nanomaterials. 2013, 2013, 1-12. http://dx.doi.org/10.1155 /2013/148578.

[2] Brown, P.K.; Qureshi, A.T.; Moll, A.N.; Hayes, D.J. and Monroe, W.T. Silver nanoscale antisense drug delivery system for photoactivated gene silencing. ACS Nano., 2013, 7(4), 294859.

[3] Conde, J.; Ambrosone, A.; Sanz, V.; Hernandez, Y.; Marchesano, V.; Tian, F.; Child, H.; Berry, C. C.; Ibarra, M. R.; Baptista, P. V.; Tortiglione, C. and Fuente, J. M. Design of Multifunctional Gold Nanoparticles for In Vitro and In Vivo Gene Silencing. ACS Nano., 2012, 6 (9), 83168324.

[4] Ding, Y.; Jiang, Z,; Saha, K.; Kim, C. S.; Kim, S. T.; Landis, R. F. and Rotello, V. M. Gold Nanoparticles for Nucleic Acid Delivery. Mol Therapy., 2014, 22(6), 1075-1083. doi: $10.1038 / \mathrm{mt} .2014 .30$

[5] Dong, H.; Dai, W.; Ju, H.; Lu, H.; Wang, S.; Xu, L.; Zhou, S.F.; Zhang, Y. and Zhang, X. Multifunctional Poly(L-lactide)-Polyethylene Glycol-Grafted Graphene Quantum Dots for Intracellular MicroRNA Imaging and Combined Specific-Gene-Targeting Agents Delivery for Improved Therapeutics. ACS Appl. Mater. Interfaces., 2015, 7, 11015-11023. DOI: 10.1021/acsami.5b02803.

[6] Germershaus, O. and Nultsch, K. Localized, non-viral delivery of nucleic acids: Opportunities, challenges and current strategies. Asian J Pharma Sci., 2015, 10(3), 159-175.

[7] Hossain, U and Kojima, C. Dendrimers for theranostic applications. Biomol Concepts. 2015, 6(3), 205-217. DOI: $10.1515 / \mathrm{bmc}-2015-0012$.

[8] Hou, W.; Wen, S.; Guo, R.; Wang, S. and Shi, X. Partially Acetylated Dendrimer-Entrapped Gold Nanoparticles with Reduced Cytotoxicity for Gene Delivery Applications. J Nanosci Nanotechnol., 2015, 15(6), 4094-4105.

[9] Huang, Y.; Lee, H.; Tolliver, L. M. and Aronstam R. S. Delivery of Nucleic Acids and Nanomaterials by Cell-Penetrating Peptides: Opportunities and Challenges. BioMed Res Int., 2015, 2015,16, Article ID 834079

[10] Huo, S.; Jin, S.; Ma, X.; Xue, X.; Yang, K.; Kumar, A.; Wang, P. C.; Zhang, J.; Hu, Z. and Liang, X. Ultrasmall Gold Nanoparticles as Carriers for Nucleus-Based Gene Therapy Due to Size-Dependent Nuclear Entry. ACS Nano., 2014, 8 (6), 5852-5862.

[11] Jin, L.; Zeng, X; Liu, M.; Deng, Y and He, N. Current Progress in Gene Delivery Technology Based on Chemical Methods and Nano-carriers. Theranostics, 2014, 4(3), 240-255.

[12] Katragadda, C.S.; Choudhury, P.K. and Murthy, P.N. Nanoparticles as Non-Viral Gene Delivery Vectors. Indian J. Pharm. Educ. Res., 2010, 44(2), 109-120.

[13] Kim, E.Y.; Schulz, R.; Swantek, P.; Kunstman, K., Malim, M. H. and Wolinsky S. M. Gold nanoparticle-mediated gene delivery induces widespread changes in the expression of innate immunity genes. Gene Therapy., 2011, 2011, 1-7.

[14] Lu, W.; Wang, L.; Li, L.; Zhao, Y.; Zhou, Z.; Shi, J.; Zuo, X. and Pan, D. Quantitative investigation of the poly-adenine DNA dissociation from the surface of gold nanoparticles. Scientific Reports, 2015, 5, 10158. doi:10.1038/serp10158.

[15] Marin, S.; Vlasceanu, G.M.; Tiplea, R.E.; Bucur, I.R.; Lemnaru, M.; Marin, M.M. and Grumezescu, A.M. Applications and toxicity of silver nanoparticles: a recent review. Curr Top Med Chem., 2015, 15(16), 1596-604. 
[16] Mieszawska, A. J.; Mulder, W. J. M.; Fayad, Z. A. and Cormode, D. P. Multifunctional Gold Nanoparticles for Diagnosis and Therapy of Disease. Mol. Pharm., 2013, 10 (3), 831-847.

[17] Pathak, A.; Patnaik, S. and Gupta, K.C. Recent trends in non-viral vector-mediated gene delivery. Biotechnol. J., 2009, 4, 1559-1572.

[18] Ramamoorth, M. and Narvekar, A. Non Viral Vectors in Gene Therapy- An Overview. J Clin Diagn Res., 2015, 9(1), 01-06.

[19] Saini, V.; Martyshki, D. V.; Towner, V. D.; Mirov, S. B. and Everts, M. Limitations of Adenoviral Vector-Mediated Delivery of Gold Nanoparticles to Tumors for Hyperthermia Induction. Open Nanomed J., 2014, 2.

[20] Sarkar, K.; Banerjee, S. L.; Kundu, P. P.; Madras, G. and Chatterjee, K. Biofunctionalized surface-modified silver nanoparticles for gene delivery. J. Mater. Chem. B., 2015, 3, 5266-5276.

[21] Sironmani T. A. Comparison of nanocarriers for gene delivery and nanosensing using montmorillonite, silver nanoparticles and multiwalled carbon nanotubes. Applied Clay Sci., 2015, 103, 55-61.

[22] Suna, N. F.; Liua, Z.; Huanga, W.; Tiana, Ai-Ling. And Hua, San-Yua. The research of nanoparticles as gene vector for tumor gene therapy. Critical Rev Oncol/Hemat. 2014, 89(3), 352-357.

[23] Tiwari, P. M.; Vig, K.; Dennis V. A. and Singh S. R. Functionalized Gold Nanoparticles and Their Biomedical Applications. Nanomaterials., 2011, 1, 31-63.

[24] Uthaman, S.; Moon, M. J.; Lee, D.; Kim, W. J. and Park IK. Di-Sulfide Linked Polyethylenimine Coated Gold Nanoparticles as a Non-Viral Gene Delivery Agent in NIH-3T3 Mouse Embryonic Fibroblast. J. Nanosci Nanotechol., 2015, 15(10), 7895-7899.

[25] Yah C. S. The toxicity of Gold Nanoparticles in relation to their physiochemical properties. Biomed Res., 2013, 24 (3), 400-413. 